\title{
Atorvastatin downregulates HSP22 expression in an atherosclerotic model in vitro and in vivo
}

\author{
QI CHEN" ${ }^{*}$,JIAN XIANG ${ }^{*}$, REN GONG, HAI-YANG FANG, CONG-CONG XU, \\ HONG-ZHOU ZHANG and YAN-QING WU
}

\author{
Department of Cardiology, The Second Affiliated Hospital of Nanchang University, \\ Nanchang, Jiangxi 330006, P.R. China
}

Received May 4, 2016; Accepted June 7, 2018

DOI: $10.3892 / \mathrm{ijmm} .2018 .4015$

\begin{abstract}
One of the pathological functions of heat shock protein 22 (HSP22) is the association with inflammatory diseases and atherosclerosis. However, the effects of a high-fat diet (HFD) or oxidized low-density lipoprotein (ox-LDL) combined with atorvastatin (ATV) on HSP22 expression are entirely unknown. The present study investigated the effects of ATV on HSP22 expression in HFD-induced atherosclerotic apolipoprotein E-deficient $\left(\mathrm{ApoE}^{-/}\right)$mice and in ox-LDL-induced human umbilical vein endothelial cells (HUVECs). Furthermore, the influence of HSP22-knockdown on the HFD- or ox-LDL-induced atherosclerotic model was also examined. It was found that HFD or ox-LDL treatment significantly increased HSP22 expression in the serum and aorta, accompanied by decreased phosphorylated (p)-endothelial nitric oxide synthase (p-eNOS) activity and activated p38 mitogen-activated protein kinase (MAPK). However, these effects were suppressed by treatment with ATV. Furthermore, HSP22-knockdown showed reduced ox-LDL-induced lesions, evidenced by increased p-eNOS activity and inactivated p38 MAPK, while suppression of cell proliferation inhibition and cell cycle arrest were also observed. Taken together, the results of this study suggest that HFD or ox-LDL increased the expression of HSP22 and p-p38 MAPK, and decreased the p-eNOS activity in vitro and in vivo, and ATV could reduce the effects by downregulating HSP22 expression.
\end{abstract}

\section{Introduction}

Heat shock protein (HSP) is a highly conserved protein synthesized by organisms in response to stress. According to the

Correspondence to: Professor Yan-Qing Wu, Department of Cardiology, The Second Affiliated Hospital of Nanchang University, 1 Minde Road, Nanchang, Jiangxe 330006, P.R. China

E-mail: yanqingw_phd@sina.com

*Contributed equally

Key words: atorvastatin, heat shock protein 22, oxidized low-density lipoprotein, endothelial nitric oxide synthase, p38 MAPK molecular size, it can be divided into HSP100, HSP90, HSP70, HSP60 and small heat shock protein. HSP has been shown to serve an important role in the development and progression of atherosclerosis (AS). HSP27 exerts its anti-atherosclerotic effect by restraining the antioxidant stress reaction, reducing the inflammatory response and inhibiting the proliferation, migration of vascular smooth muscle (1). HSP60 causes inflammation in AS by increasing the endothelial dysfunction via induction of the anti-HSP60 adaptive immune reaction (2).

HSP22, a type of small molecular weight HSP (3), was first found in HeLa and melanoma cells. The HSP can be activated by different proteases and has molecular chaperone and autokinase bioactivities. HSP22 protects the cells by regulating proliferation and migration, and inhibiting apoptosis (4). Marunouchi et al (5) reported that the expression of HSP22 in cardiomyocytes was increased on the compensation stage of heart failure following myocardial infarction, and that HSP22 can protect the mitochondrial function. A decrease in the phosphorylation of HSP22 was positively correlated with mitochondrial hypofunction, which resulted in heart failure, suggesting a protective role of HSP22 in cardiomyocytes (6). However, the association between HSP22 and AS remains unclear.

Statins have often been used in AS treatment due to their pleiotropic effects on inflammation (7). Atorvastatin (ATV) exerts its anti-atherosclerotic effects by targeting the receptor for advanced glycation end products in human umbilical vein endothelial cells (HUVECs) and in Goto Kakisaki rats (8), or by downregulating HSP22 expression induced by oxidized low-density lipoprotein (ox-LDL) in HUVECs (9). In addition, statins have a number of other effects, including anti-inflammation, anti-oxidative stress and improving endothelial function (10). However, the specific mechanism of ATV on AS and the effect of ATV on HSP22 expression remain unknown.

The present study aimed to investigate whether ATV exerts part of its inhibitory role on the progression of AS by targeting HSP22. Specifically, the expression of HSP22 and its downstream p38 signaling, and endothelial nitric oxide synthase (eNOS) activity in apolipoprotein E-deficient $\left(\mathrm{ApoE}^{-/}\right)$ mice and HUVECs. Moreover, cell proliferation and the cell cycle were also measured in HUVECs with HSP22 knockdown by shRNA transfection. 


\section{Materials and methods}

Animals and diets. A total of 36 male $\mathrm{ApoE}^{-1-}$ mice (8 weeks old, 18-22 g), provided by the Scientific Research Institute (Shanghai, China), were housed using a $12 \mathrm{~h}$ light/dark cycle at a constant temperature of $25^{\circ} \mathrm{C}$, with a relative humidity of $60-70 \%$. The mice were randomly divided into three groups: The normal diet group (ND; 12 mice), the high-fat diet group (HFD; 12 mice) (diets both from Mediscience Ltd., Jiangsu, China) and the HFD plus ATV group (HFD + ATV; 12 mice; ATV from Pfizer, Inc., Suzhou, China). Mice in the ATV-treated group were treated with $10 \mathrm{mg} / \mathrm{kg} / \mathrm{day}$ ATV via intragastric administration. All the mice were fed for 13 weeks with an HFD, and otherwise were treated with ATV for 9 weeks subsequent to being fed for 4 weeks with an HFD. Subsequent to being fed with HFD or ATV, mice were further fed with a normal diet for 1 week and anesthetized with $3 \%$ sodium pentobarbital $(40 \mathrm{mg} / \mathrm{kg}$; Sigma-Aldrich; Merck KGaA, Darmstadt, Germany) via intraperitoneal injection prior to cervical dislocation. All animal care and experimental procedures in the current study complied with the protocol approved by the Second Affiliated Hospital of Nanchang University (Nanchang, China).

Metabolic profile analysis. Serum collected from the blood samples of the three different groups was used to measure levels of plasma lipids, including total cholesterol (TC), triglycerides (TG), low-density lipoprotein cholesterol (LDL) and high-density lipoprotein cholesterol (HDL), using a cholesterol kit (cat. no. 000060094), triglycerides kit (cat. no. 000060104), HDL-cholesterol kit (cat. no. 000000190) and LDL-cholesterol kit (cat. no. 000000210) (all BioSino Biotechnology Co., Ltd., Shanghai, China), respectively.

Immunohistochemistry. Aortas of the three different groups were collected and quickly frozen in liquid nitrogen, as previously described (8), and then stored at $-80^{\circ} \mathrm{C}$ for hematoxylin and eosin (H\&E) and immunohistochemical studies, as previously described $(11,12)$. Paraffin-embedded aorta sections (4 to $7-\mu \mathrm{m}$ thick) were fixed in $10 \%$ formalin overnight at room temperature, and separately dehydrated by 50, 70, 85, 95 and $100 \%$ ethanol for $2 \mathrm{~h}$, then deparaffinized by dimethylbenzene for $15 \mathrm{~min}$ and separately hydrated by $100,95,85$ and $75 \%$ ethanol for $5 \mathrm{~min}$ at room temperature. Following blocking with $2 \%$ bovine serum albumin (Sigma Aldrich; Merck KGaA) in PBS for $1 \mathrm{~h}$ at room temperature, the tissue sections were incubated with anti-HSP22 (1:100 dilution; cat. no. ab151552; Abcam, Cambridge, MA, USA) and anti-phosphorylated p38 (anti-p-p38) (1:1,600; cat. no. 9212; Cell Signaling Technology, Inc., Danvers, MA, USA) primary antibodies at $4^{\circ} \mathrm{C}$ overnight, and then incubated with biotinylated secondary antibodies, horseradish peroxidase goat anti-rabbit $\operatorname{IgG}(1: 100$ dilution; cat. no. 111-035-008) and goat anti-mouse $\operatorname{IgG}(\mathrm{H}+\mathrm{L})$ (1:100 dilution; cat. no. 111-035003) (noth Jackson ImmunoResearch, West Grove, PA, USA) for $1 \mathrm{~h}$ at room temperature. Five fields from each slide were examined using microscopy (x200 magnification) and images were captured using a light microscope equipped with a camera (Olympus BX-50; Olympus Corporation, Tokyo, Japan).

Enzyme-linked immunosorbent assay (ELISA). Secretion of HSP22 from serum samples of the three different groups was determined by ELISA using the Heat Shock 22kDa Protein 8 (HSPB8) ELISA kit (cat. no. ABIN425260) according to the manufacturer's protocols (Beijing 4A Biotech Co., Ltd., Beijing, China).

Cell culture. HUVECs were purchased from Eidia Ltd.; Sekisui Chemical Co., Ltd. (Tokyo, Japan) and cultured in The Second Affiliated Hospital of Nanchang University. HUVECs at passage 3-4 were used for experiments. The cells were cultured with serum-free RPMI-1640 medium for $24 \mathrm{~h}$ prior to treatment. The HUVECs were randomly assigned to the indicated groups as follows: The control group, the ox-LDL $(160 \mu \mathrm{g} / \mathrm{ml})$ group, the ATV $(40 \mu \mathrm{M})$ group and the ox-LDL $(160 \mu \mathrm{g} / \mathrm{ml})$ plus ATV $(40 \mu \mathrm{M})$ group. HUVECs were treated with ox-LDL or ATV alone for $24 \mathrm{~h}$. For the combined group, HUVECs were treated with ox-LDL for $24 \mathrm{~h}$, followed by ATV for $24 \mathrm{~h}$. Cells were cultured at $37^{\circ} \mathrm{C}$ for $24 \mathrm{~h}$ with $5 \% \mathrm{CO}_{2}$.

Short hairpin RNA (shRNA) transfection. HSP22 shRNA-1, 5'-GCTGGGAGCCTGTCAGTTTAT-3'; HSP22 shRNA-2, 5'-GGATCCTGTGACAGTATTTGC-3'; and HSP22 shRNA-3, 5'-GCAGTTTCAACAACGAGCTTC-3', designed for targeting human SLC44A5 mRNA, were cloned into a lentiviral vector (pLKO.1-EGFP; Addgene, Cambridge, MA, USA). The cells were transfected with pLKO.1-EGFP-HSP22-shRNA (40 nM) using Lipofectamine 2000 (Thermo Fisher Scientific, Inc., Waltham, MA, USA) according the manufacturer's protocol. A non-specific scramble shRNA sequence was used as negative control (NC; 5'-TTCTCCGAACGTGTCACGT-3'). Cell proliferation and the cell cycle were analyzed at $48 \mathrm{~h}$ post-transfection.

MTT assay. HUVECs were treated with ox-LDL $(20,40,80$, 160 and $320 \mu \mathrm{g} / \mathrm{ml})$ or ATV $(20,40,80,160$ and $320 \mu \mathrm{M})$, respectively, while HUVECs with HSP22 shRNA transfection were treated with ox-LDL $(160 \mu \mathrm{g} / \mathrm{ml})$, ATV $(40 \mu \mathrm{M})$ or ox-LDL $(160 \mu \mathrm{g} / \mathrm{ml})$ plus ATV $(40 \mu \mathrm{M})$, and incubated for 24 , 48 and $72 \mathrm{~h}$. Next, the HUVECs were cultured with $20 \mu \mathrm{l}$ MTT $(5 \mathrm{mg} / \mathrm{ml})$ for $4 \mathrm{~h}$. The intracellular formazan crystals formed were solubilized with acidic isopropanol (Sigma-Aldrich; Merck KGaA) and the absorbance was read on a microplate reader (Utrao Medical Instrument, Shanghai, China) at $570 \mathrm{~nm}$.

Cell cycle assay. HUVECs with HSP22 shRNA transfection $\left(5 \times 10^{5}\right.$ cells/well) were treated with ox-LDL $(160 \mu \mathrm{g} / \mathrm{ml})$, ATV $(40 \mu \mathrm{M})$ or ox-LDL $(160 \mu \mathrm{g} / \mathrm{ml})$ plus ATV $(40 \mu \mathrm{M})$, and subsequently incubated with propidium iodide (PI) and $0.5 \mu \mathrm{g} / \mu \mathrm{l}$ RNase A for $30 \mathrm{~min}$. Thereafter, the cells were analyzed on a flow cytometer (BD Biosciences, San Diego, CA, USA).

$m R N A$ quantization by reverse transcription-quantitative polymerase chain reaction ( $R T-q P C R)$. Total RNA isolated from aortas or HUVECs using TRIzol and purified with an RNAeasy kit (both Thermo Fisher Scientific, Inc.) was reversed transcribed to cDNA using the Prime-Script RT reagent kit (Takara Bio, Inc., Otsu, Japan). RT-qPCR was performed with the ABI 7500 (Applied Biosystems, Foster City, CA, USA) using SYBR Premix Ex Taq (Takara Bio). Primers were as follows: HSP22 sense, 5'-CAGGTCCCTCCTTACTCA-3' and antisense, 5'-CCCGCACCCTCTAACAT-3'; and $\beta$-actin sense, 5 '-AGG 

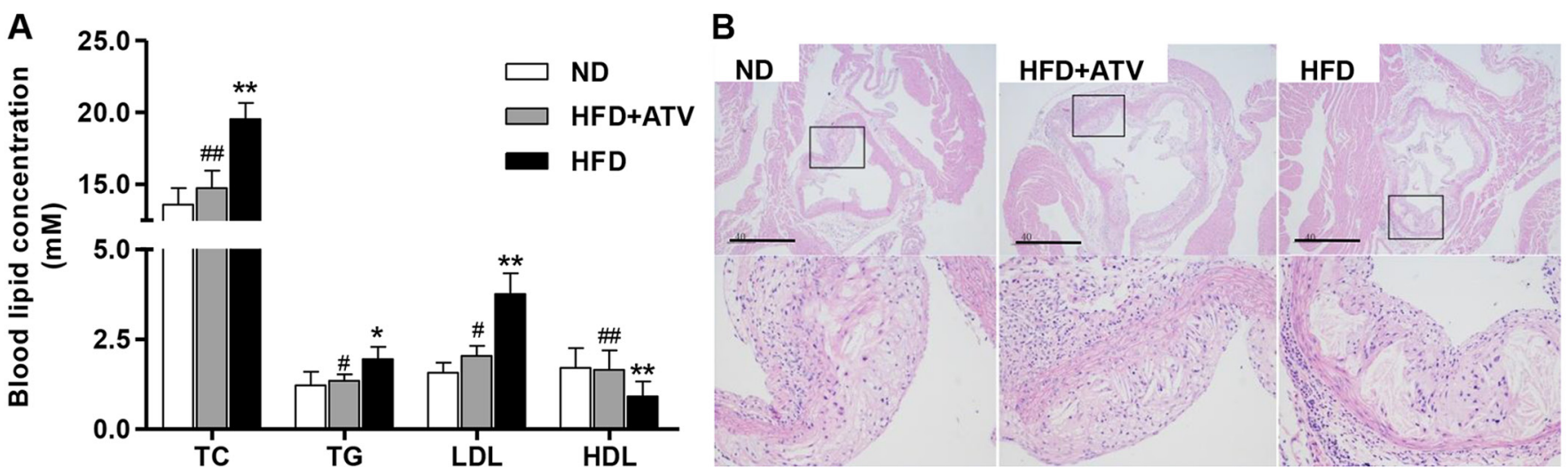

Figure 1. Atherosclerotic lesion formation in aortic sections from $\mathrm{ApoE}^{-/}$mice. (A) There were significantly increased TC, TG and LDL levels, and decreased HDL levels in the mice in the HFD group. (B) Atherosclerotic lesions in the aortic root, as measured by hematoxylin and eosin staining. Data are presented as the mean \pm standard deviation $(\mathrm{n}=6)$. ${ }^{*} \mathrm{P}<0.05$ and ${ }^{* *} \mathrm{P}<0.01$ compared with the ND group; ${ }^{\sharp} \mathrm{P}<0.05$ and ${ }^{\# \#} \mathrm{P}<0.01$ compared with the HFD group. Scale bars, $40 \mu \mathrm{m}$. ND, normal diet; HFD, high-fat diet; $\mathrm{ApoE}^{-/}$, apolipoprotein E-deficient; TC, total cholesterol; TG, triglycerides; LDL, low-density lipoprotein cholesterol; HDL, high-density lipoprotein cholesterol; ATV, atorvastatin.

GGCCGGACTCGTCATACT-3' and antisense, 5'-GGCGGC ACCACCATGTACCCT-3'. The HSP22 mRNA level was normalized by internal $\beta$-actin mRNA. The following thermocycling conditions were used for the PCR: $95^{\circ} \mathrm{C}$ for $10 \mathrm{~min}$, followed by 40 cycles of $95^{\circ} \mathrm{C}$ for $15 \mathrm{sec}$ and $60^{\circ} \mathrm{C}$ for $45 \mathrm{sec}$, and a final extension step of $95^{\circ} \mathrm{C}$ for $15 \mathrm{sec}, 60^{\circ} \mathrm{C}$ for $1 \mathrm{~min}, 95^{\circ} \mathrm{C}$ for $15 \mathrm{sec}$ and $60^{\circ} \mathrm{C}$ for $15 \mathrm{sec}$. The relative quantification values for the gene expression were calculated using the $2^{-\Delta \Delta \mathrm{Cq}}$ method (13).

Western blot analysis. Total protein was extracted from aortas or HUVECs using radioimmunoprecipitation buffer (JRDUN Biotechnology Co., Ltd. Shanghai, China). The total protein concentration in each sample was measured using a Lowry protein assay kit (Bio Rad Laboratories, Inc., Hercules, CA, USA). Equal amounts of extracted protein $(50 \mu \mathrm{g})$ were separated by SDS-PAGE on a $10 \%$ gel and transferred onto polyvinylidene difluoride membranes (Roche Diagnostics GmbH, Mannheim, Germany), followed by blocking in 5\% skimmed milk overnight at $4^{\circ} \mathrm{C}$. The protein abundance was detected with antibodies against HSP22 (1:1,000 dilution; cat. no. ab151552; Abcam), p-eNOS [1:500 dilution; cat. no. YS-(kt)-0446; YS Biotechnology Co., Ltd., Shanghai, China], eNOS (1:1,000 dilution; cat. no. ab76198; Abcam), p-p38 (1:1,000 dilution; cat. no. 4511), p38 (1:1,000 dilution; cat. no. 9212), anti-GAPDH (1:1,500; cat. no. 5174) and anti- $\beta$-actin (1:1,000; cat. no. 4970) (all Cell Signaling Technology, Inc.) for $2 \mathrm{~h}$ at room temperature. Next, the membranes were incubated with the aforementioned fluorescence secondary antibodies for $1 \mathrm{~h}$ at $37^{\circ} \mathrm{C}$. Chemiluminescence detection was conducted using Western Lightning Chemiluminescence Reagent Plus (PerkinElmer, Inc., Waltham, MA, USA). and signal intensity was determined using ImageJ software version 1.46 (National Institutes of Health, Bethesda, MD, USA).

Statistical analysis. The data are presented as the mean \pm standard deviation of at least two independent studies each performed in triplicate. Statistical analyses were performed using GraphPad Prism 5.0 (GraphPad Software, Inc., La Jolla, CA, USA). Comparisons of means between groups were analyzed using one way analysis of variance followed by Tukey's post hoc test. $\mathrm{P}<0.05$ was considered to indicate a statistically significant difference.

\section{Results}

ATV reduces atherosclerotic lesion formation in Apo $E^{-1-}$ mice. Fig. 1A shows the data on the TC, TG, LDL and HDL levels of the $\mathrm{ApoE}^{-/-}$mice. Statistical analysis showed that HFD treatment for 12 weeks significantly increased the levels of TC, TG and LDL compared with the ND group, with HDL as an exception. However, the data indicated that ATV can markedly reduce the effects of HF on the levels of TC, TG, LDL and HDL in $\mathrm{ApoE}^{-/}$mice. Moreover, the $\mathrm{H} \& \mathrm{E}$ staining results of aortic root showed the presence of more atherosclerotic plaques in the $\mathrm{ApoE}^{-/-}$mice with HFD treatment compared with that in the ND group (Fig. 1B). Whereas, in $\mathrm{ApoE}^{-/}$mice, the ATV treatment showed fewer atherosclerotic plaques than in HFD group. These data show that ATV can inhibit the formation of atherosclerotic areas in the aortic roots of $\mathrm{ApoE}^{-/}$mice.

ATV alters the content of HSP22, p-eNOS and p-p38 in atherosclerotic lesions. Aorta cross-section immunochemistry results showed that there was significantly less HSP22 in the aortic plaques following intervention with $\mathrm{ATV}$ in $\mathrm{ApoE}^{-1-}$ mice when compared with HFD treatment alone (Fig. 2A). The similar effects of HFD and ATV treatment on the content of HSP22 were also found at the serum (Fig. 2B) and aortic tissue (Fig. 2C and D) levels. In addition, compared with the ND group, the HFD group exhibited decreased p-eNOS expression in the aortic tissue (Fig. 2C and E), while the p-p38 increased (Fig. 2C, E and F). However, ATV did significantly augment p-eNOS and reduce p-p38 in the aortic tissue of the HFD group. These data show that ATV can activate the eNOS signaling pathway and inhibit the p38 mitogen-activated protein kinase (MAPK) signaling pathway, which have already been shown to serve a key role in anti-atherosclerotic effects (14).

Effects of ox-LDL and ATV on HUVEC proliferation. The concentration-dependent effect of ox-LDL on HUVEC proliferation is shown in Fig. 3A. As shown in Fig. 3A, compared with the ND group, HUVEC proliferation was increased with ox-LDL stimulation at lower concentration $(20 \mu \mathrm{g} / \mathrm{ml})$ at 24 and $48 \mathrm{~h}$; however, incubation with different 
A

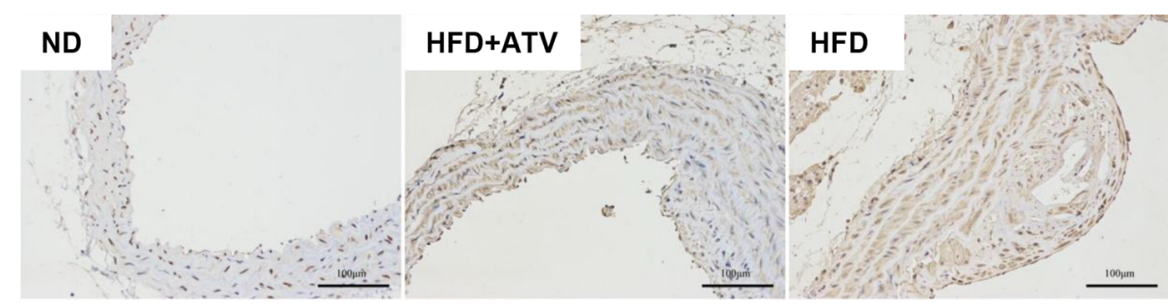

B

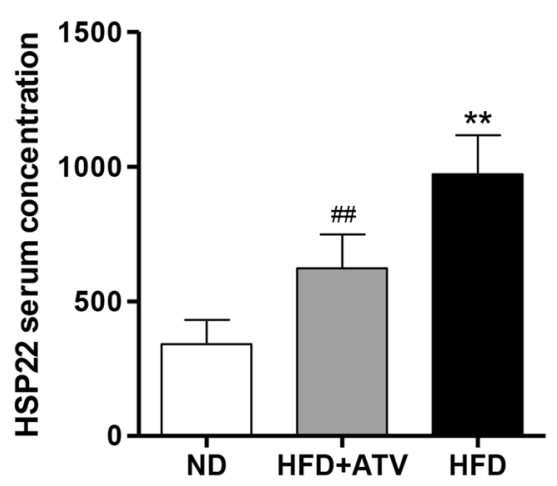

C

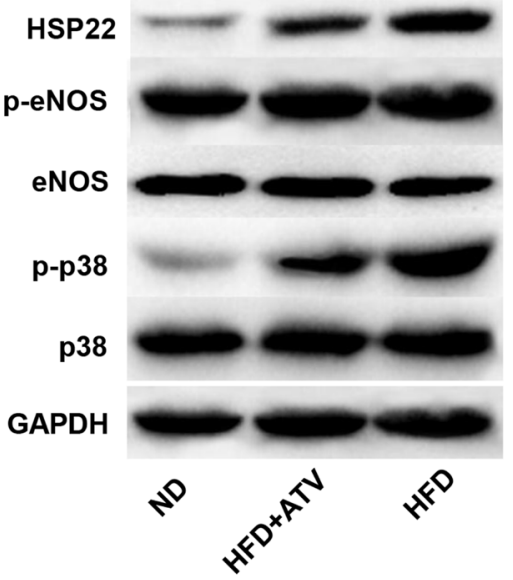

D

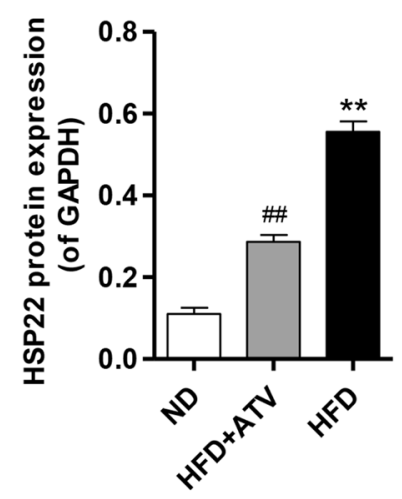

E

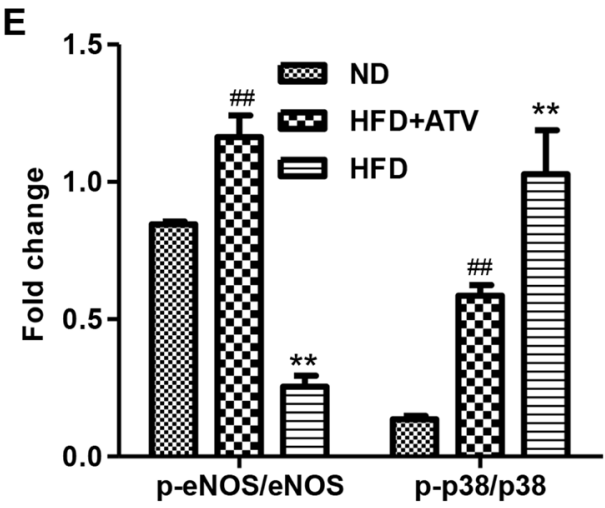

F

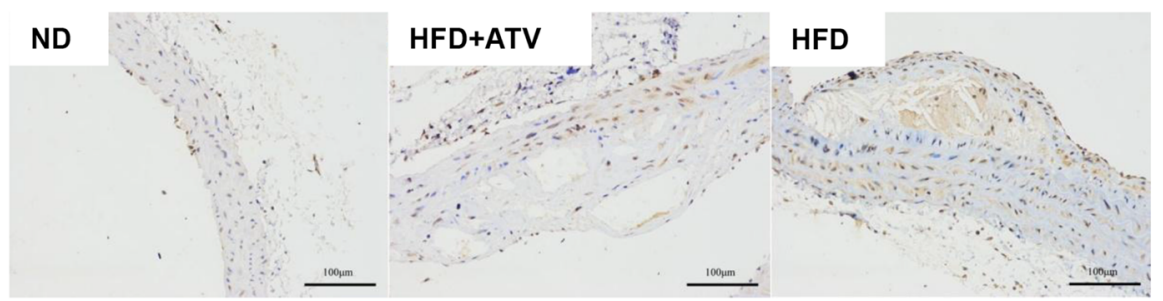

Figure 2. Effect of HFD on HSP22, eNOS and p38 MAPK in atherosclerotic lesions. The expression of HSP22 in ApoE ${ }^{-/-}$mice with an HFD and/or ATV treatment was measured by (A) immunohistochemistry, (B) enzyme-linked immunosorbent assay and (C and D) western blot assay, respectively. (C and E) The expression level of p-eNOS was examined by western blot assay in ApoE ${ }^{-/}$mice with an HFD and/or ATV treatment. The expression level of p-p38 MAPK was examined by (C and E) western blot assay and (F) immunohistochemistry in ApoE ${ }^{-/-}$mice with an HFD and/or ATV treatment. Data are presented as the mean \pm standard deviation $(\mathrm{n}=6) .{ }^{* *} \mathrm{P}<0.01$ compared with the ND group; ${ }^{\# \#} \mathrm{P}<0.01$ compared with the HFD group. Scale bars, $100 \mu \mathrm{m}$. ND, normal diet; HFD, high-fat diet; HSP22, heat shock protein 22; eNOS, endothelial nitric oxide synthase; MAPK, mitogen-activated protein kinase; ApoE ${ }^{-/}$, apolipoprotein E-deficient; ATV, atorvastatin; p-phosphorylated.

concentrations of ox-LDL (80, 160 and $320 \mu \mathrm{g} / \mathrm{ml})$ led to a significant decrease. The initial significant decrease in proliferation compared with the control was observed following incubation of HUVECs with $80 \mu \mathrm{g} / \mathrm{ml}$ ox-LDL at 24,48 and $72 \mathrm{~h}$. To investigate the effects of ATV on the cytotoxicity of HUVECs, the proliferation of HUVECs treated with ATV at different concentrations and for time periods was also measured. Stimulation of HUVECs with 40 and $80 \mu \mathrm{M}$ ATV led to a significant increase in HUVEC proliferation that was highest at $72 \mathrm{~h}$ post-ATV application, while decreased proliferation was noted when using higher concentrations of ATV treatment (160 and $320 \mu \mathrm{M})$ in HUVECs at $72 \mathrm{~h}$ (Fig. 3B). These results indicate that ox-LDL and ATV induce the proliferation of HUVECs in a concentration- and time-dependent manner. Therefore, $160 \mu \mathrm{g} / \mathrm{ml}$ ox-LDL and $40 \mu \mathrm{M}$ ATV were used for subsequent experiments. 

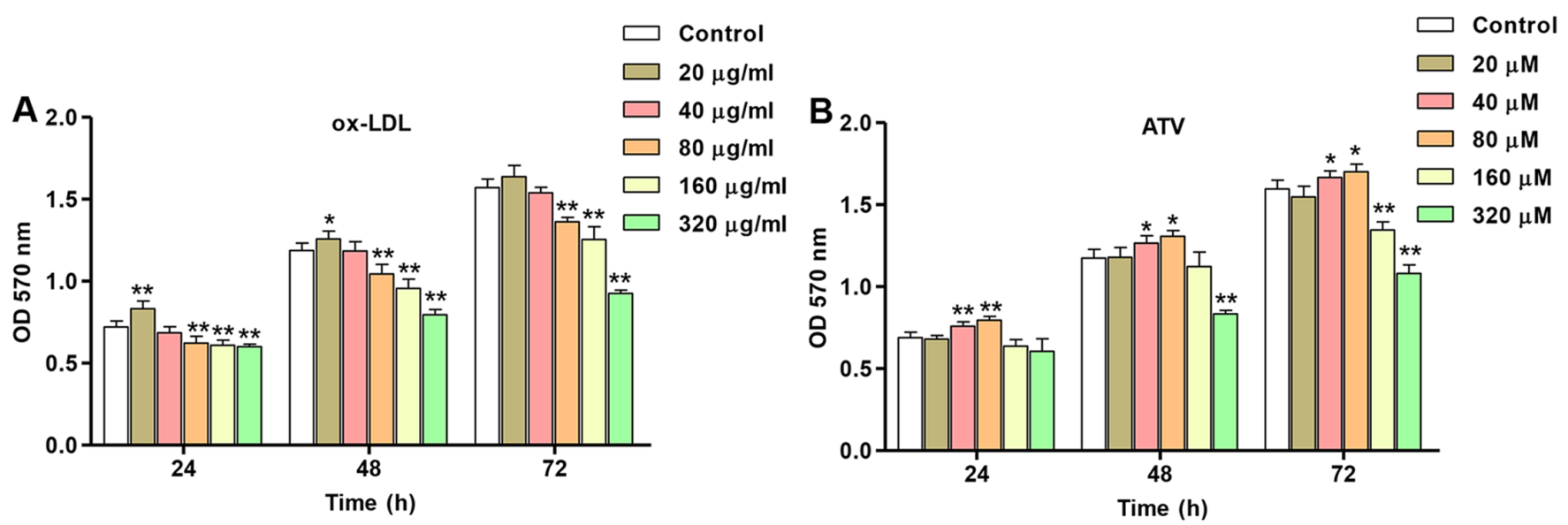

Figure 3. Effect of ox-LDL and ATV on cell proliferation of HUVECs. Cell proliferation was measured by MTT assay in HUVECs with different concentrations of (A) ox-LDL or (B) ATV treatment for 24,48 and $72 \mathrm{~h}$. ${ }^{*} \mathrm{P}<0.05$ and ${ }^{* *} \mathrm{P}<0.01$ compared with the control group. ox-LDL, oxidized low-density lipoprotein; ATV, ATV, atorvastatin; HUVECs, human umbilical vein endothelial cells; OD, optical density.

A

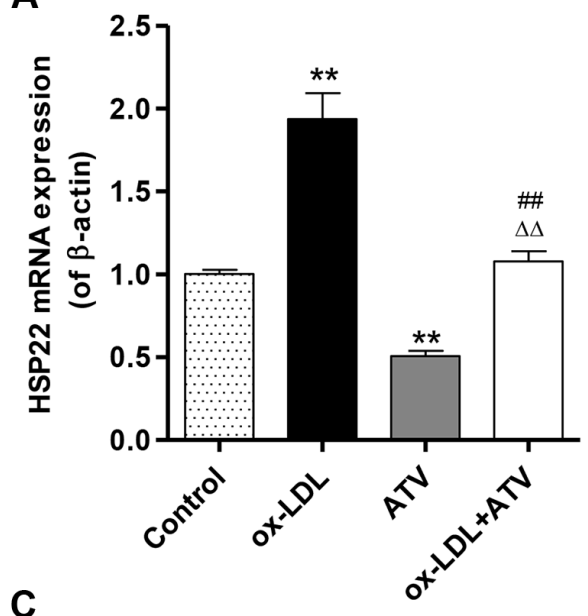

C

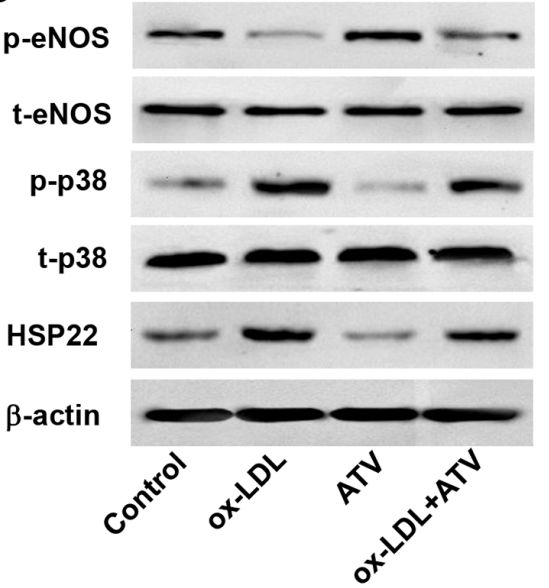

B
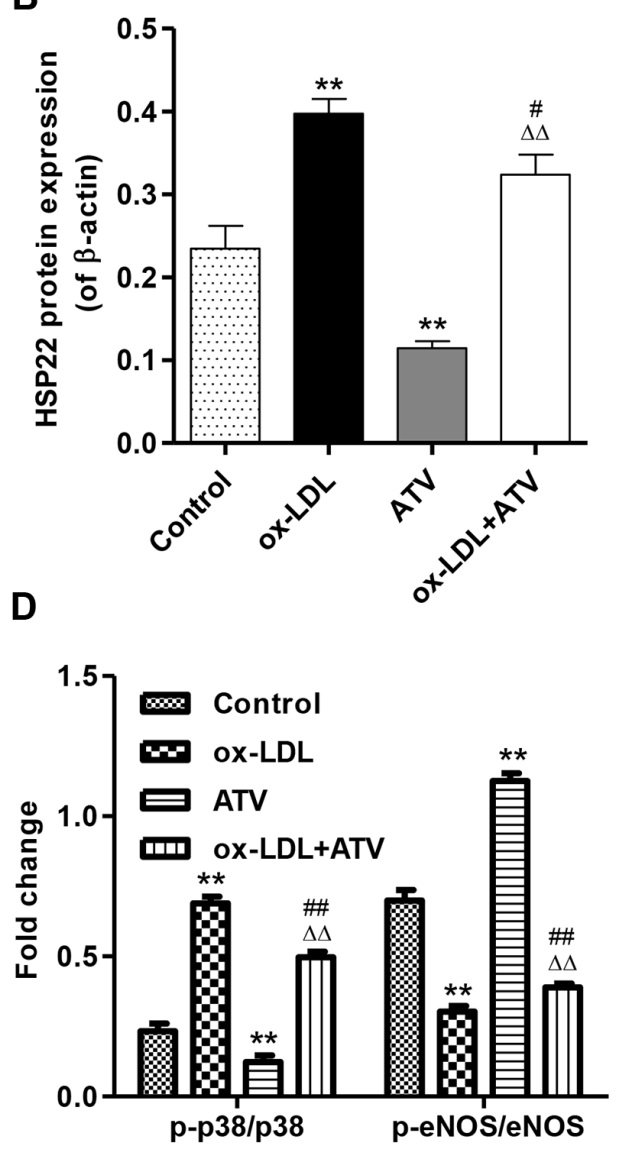

Figure 4. Effect of ATV on HSP22, eNOS and p38 MAPK in HUVECs. The expression of HSP22 in HUVECs with an HFD and/or ATV treatment was measured by (A) reverse transcription-quantitative polymerase chain reaction and (B) western blot assay, respectively. (C and D) The expression levels of p-eNOS and p-p38 MAPK were examined by western blot assay in HUVECs with an HFD and/or ATV treatment. ${ }^{* *} \mathrm{P}<0.01$ compared with the control group; ${ }^{*} \mathrm{P}<0.05$ and ${ }^{\# \#} \mathrm{P}<0.01$ compared with the ox-LDL group; ${ }^{\triangle} \mathrm{P}<0.01$ compared with the ATV group. ox-LDL, oxidized low-density lipoprotein; ATV, atorvastatin; HUVECs, human umbilical vein endothelial cells; HSP22, heat shock protein 22; eNOS, endothelial nitric oxide synthase; MAPK, mitogen-activated kinase; HFD, high-fat diet; p-, phosphorylated; t-, total.

ATV inhibits the effects of ox-LDL on the expression of HSP22, p-eNOS and p-p38 in HUVECs. Fig. 4A and B show that $160 \mu \mathrm{g} / \mathrm{ml}$ ox-LDL treatment for $24 \mathrm{~h}$ significantly increased the mRNA and protein expression of HSP22 in HUVECs compared with the control group, but that $40 \mu \mathrm{M}$ ATV treatment for $24 \mathrm{~h}$ significantly decreased HSP22 

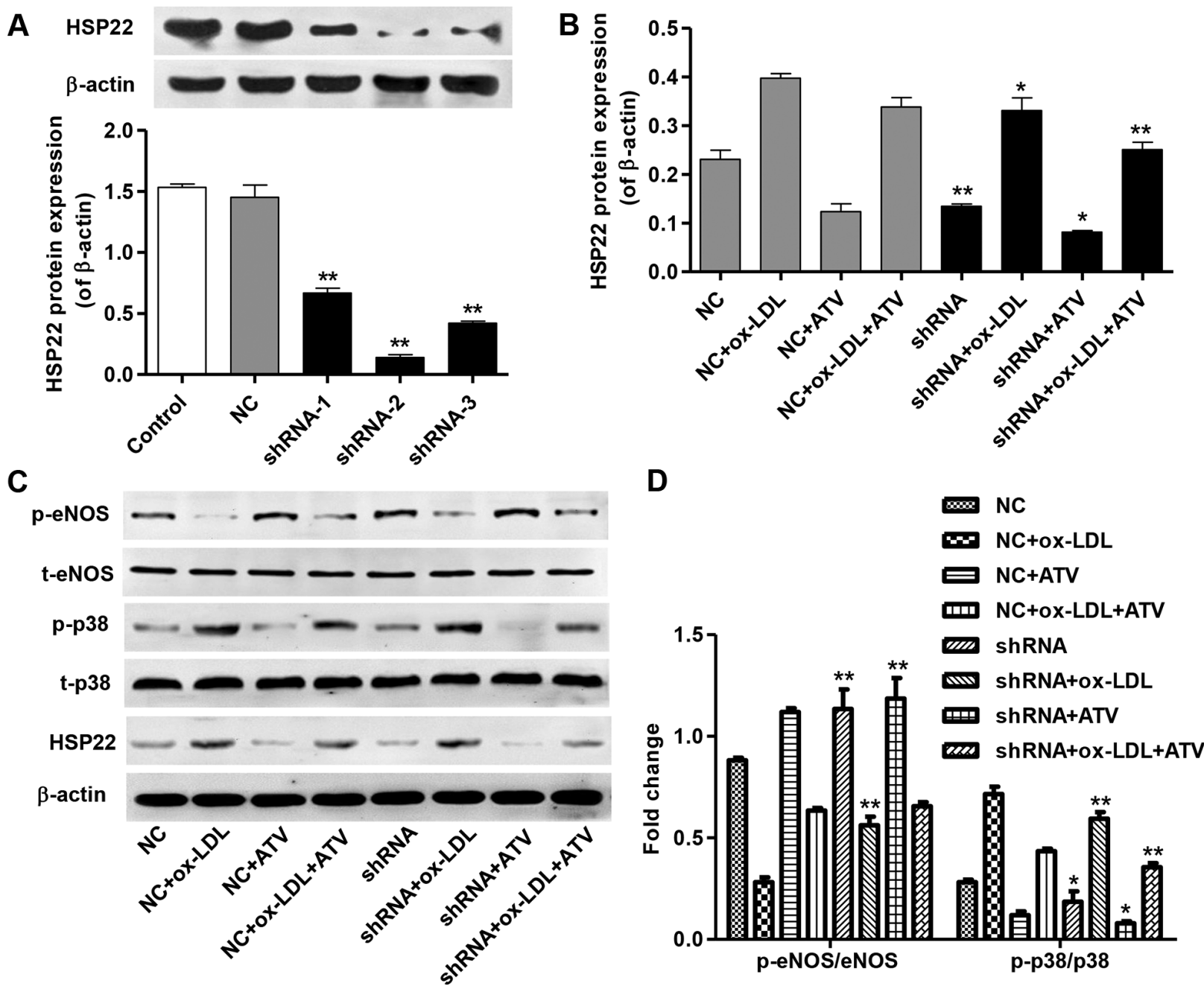

Figure 5. HSP22-knockdown inhibits ox-LDL-induced p-eNOS decrease and p-p38 increase in HUVECs. (A) The transfection efficiency of HSP22 shRNAs in HUVECs was measured by western blot assay. (B) The expression of HSP22 in HUVECs with an HFD and/or ATV treatment was measured by western blot assay. (C and D) The expression of p-eNOS and p-p38 in HUVECs with an HFD and/or ATV treatment in the absence or presence of HSP22 shRNA was measured by western blot assay. " $\mathrm{P}<0.05$ and ${ }^{* *} \mathrm{P}<0.01$ compared with the corresponding NC group. ox-LDL, oxidized low-density lipoprotein; ATV, atorvastatin; HUVECs, human umbilical vein endothelial cells; HSP22, heat shock protein 22; eNOS, endothelial nitric oxide synthase; MAPK, mitogen-activated kinase; HFD, high-fat diet; p-, phosphorylated; t-, total; NC, negative control; shRNA, short hairpin RNA.

expression. Prior to the ATV treatment, pretreatment with ox-LDL at a concentration of $160 \mu \mathrm{g} / \mathrm{ml}$ for $24 \mathrm{~h}$ significantly decreased the mRNA and protein expression of HUSP22 in HUVECs compared with the group with ox-LDL treatment alone. Fig. 4C and D show that stimulation of ox-LDL caused decreased p-eNOS and increased p-p38 levels in HUVECs compared with the control group, but that ATV had an inverse effect. Prior to the ATV treatment, pretreatment with ox-LDL significantly increased p-eNOS and decreased p-p38 levels in HUVECs compared with using the ox-LDL treatment alone.

HSP22 shRNA alters the expression of p-eNOS and p-p38 in HUVECs induced by ox- $L D L$. To elucidate whether the cytoprotection of ATV was associated with its downregulation of HSP22 in ox-LDL-stimulated HUVECs, three shRNAs targeting HSP22 were cloned into a lentiviral vector for HUSP22-knockdown. shRNA-2 showed a minimal HSP22 protein level compared with the other two shRNAs in the HUVECs, but exhibited no effect in NC-transfected HUVECs (Fig. 5A). Moreover, shRNA transfection in HUVECs significantly decreased HSP22 expression in ox-LDL, ATV and ox-LDL plus ATV treatment groups compared with corresponding NC groups (Fig. 5B). Notably, increased p-eNOS levels were found only in HUVECs with shRNA or shRNA plus ox-LDL treatment groups compared with the corresponding NC groups (Fig. 5C and D). Similarly, HSP22 shRNA markedly decreased the p-p38 levels in ox-LDL, ATV and ox-LDL plus ATV treatment groups compared with that in the corresponding $\mathrm{NC}$ groups (Fig. 5C and D). These findings suggest that the inhibition of the cytotoxicity of ATV is associated with its downregulation of HSP22.

HSP22 shRNA reduces proliferation inhibition and cell cycle arrest of HUVECs induced by ox-LDL. The effects of HSP22-knockdown on cell proliferation and the cell cycle progression of HUVECs were measured by MTT and flow cytometry analysis, respectively. ATV treatment markedly reversed the inhibition of proliferation of HUVECs with NC or HSP2 2 shRNA transfection caused by ox-LDL treatment at 24, 48 and $72 \mathrm{~h}$ (Fig. 6A-C). More importantly, HSP22 


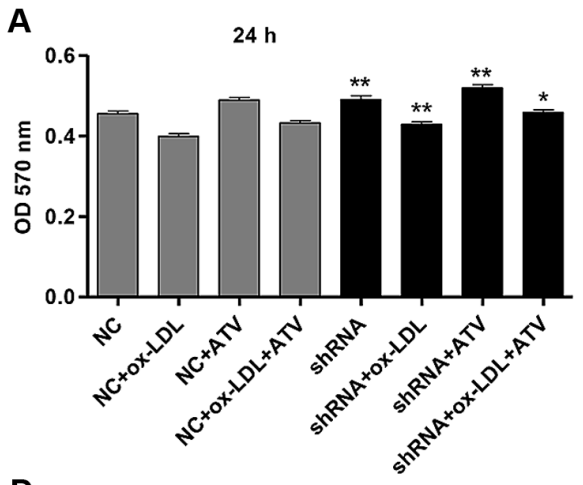

D
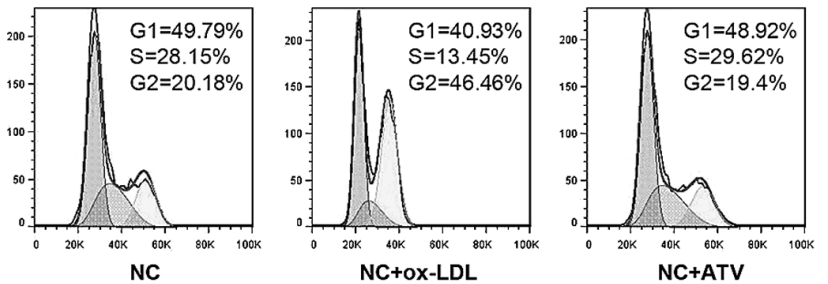

B

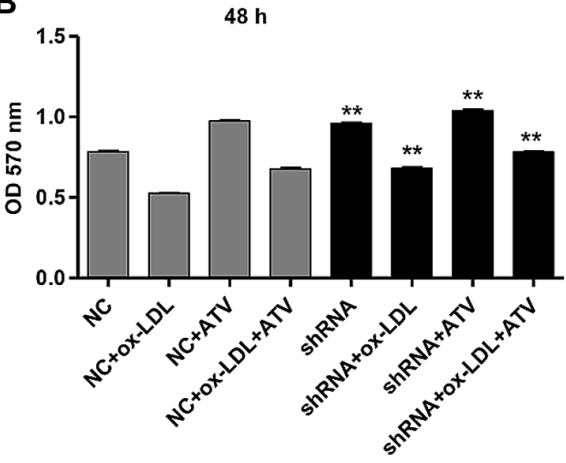

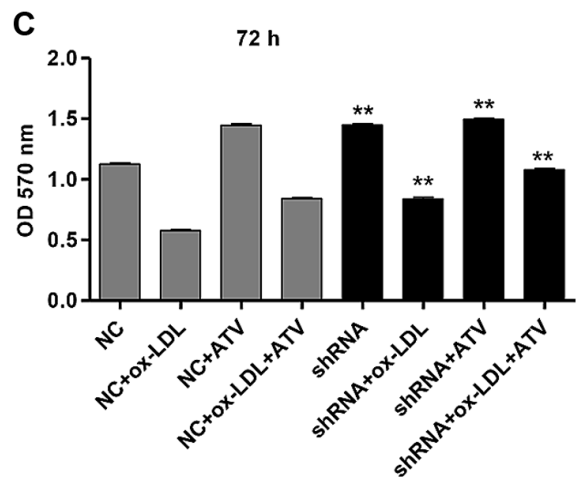

E

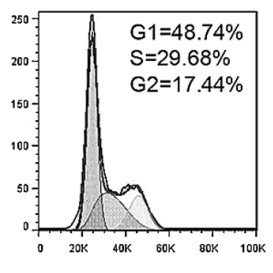

ShRNA

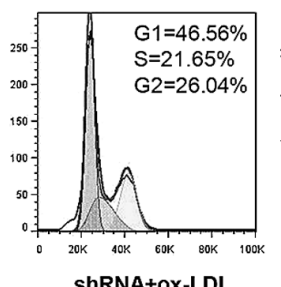

shRNA+ox-LDL

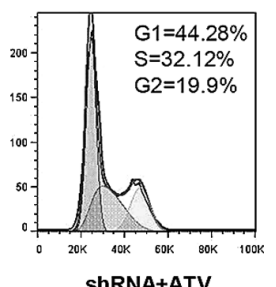

ShRNA+ATV
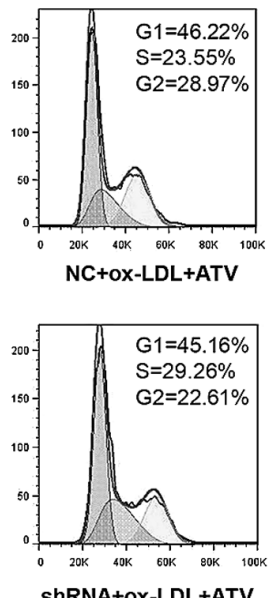

ShRNA+OX-LDL+ATV

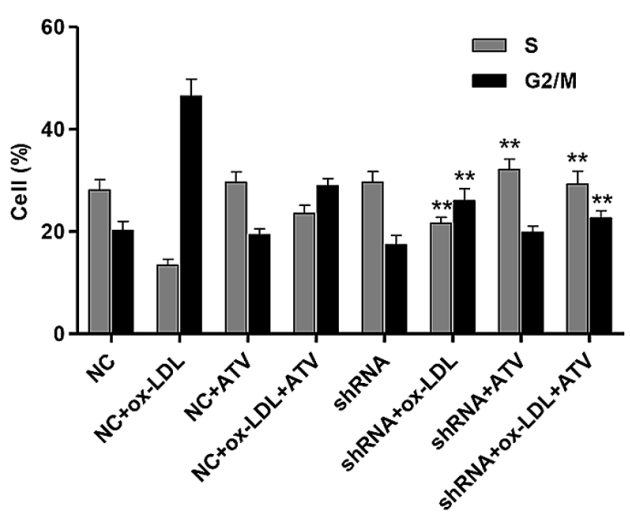

Figure 6. HSP22-knockdown reduces ox-LDL-induced cell proliferation inhibition and cell cycle arrest in HUVECs. Cell proliferation was measured by MTT assay in HUVECs with an HFD and/or ATV treatment in the absence or presence of HSP22 shRNA for (A) $24 \mathrm{~h}$, (B) $48 \mathrm{~h}$ and (C) $72 \mathrm{~h}$. (D and E) The cell cycle was measured by flow cytometry assay in HUVECs with an HFD and/or ATV treatment in the absence or presence of HSP22 shRNA. "P<0.05 and ${ }^{* *} \mathrm{P}<0.01$ compared with the corresponding NC group. ox-LDL, oxidized low-density lipoprotein; ATV, atorvastatin; HUVECs, human umbilical vein endothelial cells; HSP22, heat shock protein 22; eNOS, endothelial nitric oxide synthase; MAPK, mitogen-activated kinase; HFD, high-fat diet; p-, phosphorylated; t-, total; NC, negative control; shRNA, short hairpin RNA; OD, optical density.

shRNA had a similar effect to ATV, which showed increased proliferation of HUVECs compared with the corresponding NC groups. Furthermore, ATV or HSP22 shRNA treatment reduced the ox-LDL-induced G2/M cell cycle arrest of HUVECs (Fig. 6D and E). However, treatment with ATV alone had no effect on the cell cycle of the HUVECs.

\section{Discussion}

Coronary artery AS is the single largest killer of men and women in the world; it is the principal cause of coronary artery disease, in which atherosclerotic changes are present within the walls of the coronary arteries (15). The accumulation of lipoproteins not only damages the endothelial cells, but also modulates the expression levels of adhesive molecules and inflammatory factors, resulting in the progression of AS (16). Depending on the model used, animals fed an HFD usually develop hyperlipidemia. ATV therapy is effective in lowering the serum lipid levels in these animals, including the cholesterol and TG levels $(17,18)$. In the present study, the lipid metabolism of $\mathrm{ApoE}^{-/-}$mice was abnormal following HFD intervention, showing increased serum levels of TC, TG and LDL, and a decreased HDL serum level. Atherosclerotic plaques were also observed in HFD-treated ApoE ${ }^{-/-}$mice, which indicated that an AS mouse model had been successfully established.
HSP22 is widely distributed in a number of tissues, particularly in skeletal muscle, smooth muscle, the myocardium and the brain (19). In the present study, the expression of HSP22 was measured by western blot analysis and immunochemistry assay. It was found that the HFD model exhibited a higher level of HSP22 expression compared with the ND group in $\mathrm{ApoE}^{-/-}$mice. LDL, particularly ox-LDL, is the main cause for AS in hyperlipidemia mice. In the present study, it was also found that ox-LDL can stimulate HUVECs and then increase the expression of HSP22. HSPs can be detected in the serum of various diseases; for example, HSP70, is considered to be the predictor for acute coronary syndrome and the risk factor for cardiovascular disease (20). The present results found that the content of HSP22 in the serum of $\mathrm{ApoE}^{-/-}$mice was significantly increased following HFD treatment.

Endothelial dysfunction, a critical and initial factor for AS, is dependent on the expression of eNOS (21). Under anoxic conditions, the increased expression of HSP22 in the myocardial cells and mitochondria can upregulate the expression of mitochondrial NOS. NO generation by eNOS possesses a protective effect on cardiomyocytes through decreasing the levels of oxidative phosphorylation, reactive oxygen and the opening of mitochondrial permeability transition pores (22). In the present study, it was found that HFD or ox-LDL stimulation decreased the levels of 
p-eNOS/eNOS, in contrast to the expression of HSP22 in $\mathrm{ApoE}^{-/-}$mice and HUVECs, which was a similar result to a previous study (23).

Activation of AMP-activated protein kinase has been reported to possess anti-atherosclerotic effects by upregulating the protein kinase B/eNOS/NO signaling pathway, leading to the suppression of $\mathrm{p} 38$-mediated nuclear factor- $\mathrm{kB}$ activation and consequent suppression of downstream inflammatory responses $(24,25)$. The suppression of MAPK has also been reported to have beneficial effects on AS through inhibition of adhesion molecules and anti-inflammatory effects, as well as increase in the stability of carotid plaques $(26,27)$. In the present study, the expression of p-p38 MAPK was markedly increased in the $\mathrm{ApoE}^{-/-}$mice and the HUVECs. In the HFD-induced AS model, inhibition of p38 MAPK promotes vasculogenic cells survival, activates the downstream mitogen- and stress-activated protein kinase and the cyclic adenosine monophosphate response element binding protein, and reduces endothelial dysfunction and AS progression (28).

ATV, as the hydroxy-3-methylglutaryl-CoA reductase inhibitor, has been reported to serve a protective role in cardiovascular disease, including AS. However, the mechanisms through which ATV attenuates the progress of AS is complex and not completely understood. Notably, in the present study, ATV decreased HSP22 and p-p38 MAPK expression, and increased $\mathrm{p}$-eNOS/eNOS expression in the ApoE $^{-/-}$mice and the HUVECs. A previous study showed that ATV reduced endothelial apoptosis through suppressing advanced glycation end product-induced injury and increasing the expression of HSP70 (29), while the upregulation of HSP22 was able to promote the inflammation of rheumatoid arthritis (30). According to these findings, we propose that ATV protects against AS by directly inhibiting the expression of HSP22. In thre present study, HSP22 downregulation increased p-eNOS/eNOS and decreased p-p38 MAPK expression in the HUVECs, suggesting that ATV reduced AS through suppressing HSP22-dependent inactivation of eNOS signaling and activation of p38 MAPK, which is in line with another previous study (31). Ye (32) reported that the inhibition of HSP22 aggravated the cell apoptosis induced by ox-LDL, indicating a protective effect of HSP22 on cells through improving ox-LDL-induced lesions. However, the present results observed that inhibition of HSP22 by shRNA transfection in HUVECs reduced cell proliferation inhibition and cell cycle G2/M arrest induced by ox-LDL.

A limitation of the present study is that it could not provide direct evidence of HSP22 activating the p38 MAPK signaling pathway. The definitive mechanisms of action for ATV require further investigation in vitro by culturing primary aortic endothelial cells. In the present study, ATV was shown to suppress ox-LDL-induced HSP22 expression, which suggests that the powerful anti-atherosclerotic effects of ATV may activate eNOS signaling and inhibit p38 MAPK signaling by suppressing HFD- or ox-LDL-induced HSP22 expression. Investigation of the HSP22 expression induced by HFD- or ox-LDL may provide a clue to understanding the pathophysiology of AS, and may lead to a novel and promising therapeutic strategy.

\section{Acknowledgements}

Not applicable.

\section{Funding}

This study was supported by the National Natural Science Foundation of China (grant no. 30800467).

\section{Availability of data and materials}

All data generated or analyzed during this study are included in this published article.

\section{Authors' contributions}

QC and JX conceived and designed the experiments. RG, $\mathrm{HF}, \mathrm{CX}$ and YW performed the experiments. CX and $\mathrm{HZ}$ analyzed the data. $\mathrm{HZ}$ and $\mathrm{YW}$ contributed with regards to the reagents/materials/analysis tools. QC, JX and YW wrote the paper. All authors read and approved the final manuscript.

\section{Ethics approval and consent to participate}

All animal care and experimental procedures in the current study complied with the protocol approved by the Second Affiliated Hospital of Nanchang University.

\section{Patient consent for publication}

Not applicable.

\section{Competing interests}

The authors declare that they have no competing interests.

\section{References}

1. Cuerrier CM, Chen YX, Tremblay D, Rayner K, McNulty M, Zhao X, Kennedy CR, de BelleRoche J, Pelling AE and O'Brien ER: Chronic over-expression of heat shock protein 27 attenuates atherogenesis and enhances plaque remodeling: A combined histological and mechanical assessment of aortic lesions. PLoS One 8: e55867, 2013.

2. Almanzar G, Öllinger R, Leuenberger J, Onestingel E, Rantner B, Zehm S, Cardini B, van der Zee R, Grundtman C and Wick G: Autoreactive HSP60 epitope-specific T-cells in early human atherosclerotic lesions. J Autoimmun 39: 441-450, 2012.

3. Smith CC, Yu YX, Kulka M and Aurelian L: A novel human gene similar to the protein kinase $(\mathrm{PK})$ coding domain of the large subunit of herpes simplex virus type 2 ribonucleotide reductase (ICP10) codes for a serine-threonine PK and is expressed in melanoma cells. J Biol Chem 275: 25690-25699, 2000.

4. Li XS, Xu Q, Fu XY and Luo WS: Heat shock protein 22 overexpression is associated with the progression and prognosis in gastric cancer. J Cancer Res Clin Oncol 140: 1305-1313, 2014.

5. Marunouchi T, Abe Y, Murata M, Inomata S, Sanbe A, Takagi N and Tanonaka K: Changes in small heat shock proteins HSPB1, HSPB5 and HSPB8 in mitochondria of the failing heart following myocardial infarction in rats. Biol Pharm Bull 36: 529-539, 2013.

6. Marunouchi T, Inomata S, Sanbe A, Takagi N and Tanonaka K: Protective effect of geranylgeranylacetone via enhanced induction of HSPB1 and HSPB8 in mitochondria of the failing heart following myocardial infarction in rats. Eur J Pharmacol 730: 140-147, 2014. 
7. Pantan R, Tocharus J, Suksamrarn A and Tocharus C: Synergistic effect of atorvastatin and Cyanidin-3-glucoside on angiotensin II-induced inflammation in vascular smooth muscle cells. Exp Cell Res 342: 104-112, 2016.

8. Feng B, Xu L, Wang H, Yan X, Xue J, Liu F and Hu JF: Atorvastatin exerts its anti-atherosclerotic effects by targeting the receptor for advanced glycation end products. Biochim Biophys Acta 1812: 1130-1137, 2011.

9. Zhang M, Zhou SH, Li XP, Shen XQ, Fang ZF, Liu QM, Qiu SF and Zhao SP: Atorvastatin downregulates BMP-2 expression induced by oxidized low-density lipoprotein in human umbilical vein endothelial cells. Circ J 72: 807-812, 2008.

10. Lin CP, Chen YH, Lin WT, Leu HB, Liu TZ, Huang SL and Chen JW: Direct effect of statins on homocysteine-induced endothelial adhesiveness: Potential impact to human atherosclerosis. Eur J Clin Invest 38: 106-116, 2008.

11. Zeng Y, Li C, Guan M, Zheng Z, Li J, Xu W, Wang L, He F and Xue Y: The DPP-4 inhibitor sitagliptin attenuates the progress of atherosclerosis in apolipoprotein-E-knockout mice via AMPK- and MAPK-dependent mechanisms. Cardiovasc Diabetol 13: 32, 2014.

12. Zhang XF, Zhu J, Geng WY, Zhao SJ, Jiang CW, Cai SR, Cheng M, Zhou CY and Liu ZB: Electroacupuncture at Feishu (BL13) and Zusanli (ST36) down-regulates the expression of orexins and their receptors in rats with chronic obstructive pulmonary disease. J Integr Med 12: 417-424, 2014.

13. Livak KJ and Schmittgen TD: Analysis of relative gene expression data using real time quantitative PCR and the 2(Delta Delta C(T)) method. Methods 25: 402 408, 2001.

14. Cuerrier CM, Chen YX, Tremblay D, Rayner K, McNulty M, Zhao X, Kennedy CR, de BelleRoche J, Pelling AE and O'Brien ER: Chronic over-expression of heat shock protein 27 attenuates atherogenesis and enhances plaque remodeling: A combined histological and mechanical assessment of aortic lesions. PLoS One 8: e55867, 2013.

15. Hodis HN, Mack WJ, Azen SP, Lobo RA, Shoupe D, Mahrer PR, Faxon DP, Cashin-Hemphill L, Sanmarco ME, French WJ, et al; Women's Estrogen-Progestin Lipid-Lowering Hormone Atherosclerosis Regression Trial Research Group: Hormone therapy and the progression of coronary-artery atherosclerosis in postmenopausal women. N Engl J Med 349: 535-545, 2003.

16. Hermida $\mathrm{N}$ and Balligand JL: Low-density lipoprotein-cholesterol-induced endothelial dysfunction and oxidative stress: The role of statins. Antioxid Redox Signal 20: $1216-1237,2014$

17. Ji G, Zhao X, Leng L, Liu P and Jiang Z: Comparison of dietary control and atorvastatin on high fat diet induced hepatic steatosis and hyperlipidemia in rats. Lipids Health Dis 10: 23, 2011.

18. Paraskevas KI, Pantopoulou A, Vlachos IS, Agrogiannis G, Iliopoulos DG, Karatzas G, Tzivras D, Mikhailidis DP and Perrea DN: Comparison of fibrate, ezetimibe, low- and high-dose statin therapy for the dyslipidemia of the metabolic syndrome in a mouse model. Angiology 62: 144-154, 2011.

19. Acunzo J, Katsogiannou M and Rocchi P: Small heat shock proteins HSP27 (HspB1), $\alpha$ B-crystallin (HspB5) and HSP22 (HspB8) as regulators of cell death. Int J Biochem Cell Biol 44: $1622-1631,2012$

20. Zhang X, Xu Z, Zhou L, Chen Y, He M, Cheng L, Hu FB, Tanguay RM and Wu T: Plasma levels of Hsp70 and anti-Hsp70 antibody predict risk of acute coronary syndrome. Cell Stress Chaperones 15: 675-686, 2010 .
21. Vergnani L, Hatrik S, Ricci F, Passaro A, Manzoli N, Zuliani G, Brovkovych V, Fellin R and Malinski T: Effect of native and oxidized low-density lipoprotein on endothelial nitric oxide and superoxide production: Key role of L-arginine availability. Circulation 101: 1261-1266, 2000.

22. Laure L, Long R, Lizano P, Zini R, Berdeaux A, Depre C and Morin D: Cardiac H11 kinase/Hsp22 stimulates oxidative phosphorylation and modulates mitochondrial reactive oxygen species production: Involvement of a nitric oxide-dependent mechanism. Free Radic Biol Med 52: 2168-2176, 2012.

23. Korkmaz Y, Bloch W, Addicks K, Schneider K, Baumann MA and Raab WH: The Basal phosphorylation sites of endothelial nitric oxide synthase at serine (Ser)1177, Ser116, and threonine (Thr)495 in rat molar epithelial rests of Malassez. J Periodontol 76: 1513-1519, 2005.

24. Zhang Y, Qiu J, Wang X, Zhang Y and Xia M: AMP-activated protein kinase suppresses endothelial cell inflammation through phosphorylation of transcriptional coactivator p300. Arterioscler Thromb Vasc Biol 31: 2897-2908, 2011.

25. Ou HC, Hsieh YL, Yang NC, Tsai KL, Chen KL, Tsai CS, Chen IJ, Wu BT and Lee SD: Ginkgo biloba extract attenuates oxLDL-induced endothelial dysfunction via an AMPK-dependent mechanism. J Appl Physiol 1985 114: 274-285, 2013.

26. Zhang K, Meng X, Kong J, Liu FF, Yang JM, Gao F, Zhang Y and Zhang C: Simvastatin increases Prolyl-4-Hydroxylase $\alpha 1$ expression in atherosclerotic plaque and ox-LDL-stimulated human aortic smooth muscle cells via p38 MAPK and ERK1/2 signaling. J Mol Cell Cardiol 65: 43-50, 2013.

27. Park SH, Sung YY, Nho KJ and Kim HK: Anti-atherosclerotic effects of Polygonum aviculare L. ethanol extract in ApoE knock-out mice fed a Western diet mediated via the MAPK pathway. J Ethnopharmacol 151: 1109-1115, 2014.

28. Seeger FH, Sedding D, Langheinrich AC, Haendeler J, Zeiher AM and Dimmeler S: Inhibition of the p38 MAP kinase in vivo improves number and functional activity of vasculogenic cells and reduces atherosclerotic disease progression. Basic Res Cardiol 105: 389-397, 2010.

29. Li Y, Li J, Cui L, Lai Y, Yao Y, Zhang Y, Pang X, Wang J and Liu X: Inhibitory effect of atorvastatin on AGE-induced HCAEC apoptosis by upregulating HSF-1 protein. Int J Biol Macromol 57: 259-264, 2013

30. Roelofs MF, Boelens WC, Joosten LA, Abdollahi-Roodsaz S, Geurts J, Wunderink LU, Schreurs BW, van den Berg WB and Radstake TR: Identification of small heat shock protein B8 (HSP22) as a novel TLR4 ligand and potential involvement in the pathogenesis of rheumatoid arthritis. J Immunol 176: 7021-7027, 2006.

31. Qiu H, Lizano P, Laure L, Sui X, Rashed E, Park JY, Hong C, Gao S, Holle E, Morin D, et al: H11 kinase/heat shock protein 22 deletion impairs both nuclear and mitochondrial functions of STAT3 and accelerates the transition into heart failure on cardiac overload. Circulation 124: 406-415, 2011.

32. Ye YP: The effect of HSP22 and Atorvastatin in the injury of HUVECs induced by ox-LDL. Nanchang University, 2014.

This work is licensed under a Creative Commons Attribution-NonCommercial-NoDerivatives 4.0 International (CC BY-NC-ND 4.0) License. 